\title{
Phase behavior of two-dimensional hard rod fluids
}

\author{
Martin A. Bates and Daan Frenkel \\ FOM Institute for Atomic and Molecular Physics, Kruislaan 407, 1098 SJ Amsterdam, The Netherlands
}

(Received 3 November 1999; accepted 4 February 2000)

\begin{abstract}
Monte Carlo simulations are used to study two-dimensional hard rod fluids consisting of spherocylinders confined to lie in a plane. The phase behavior is mapped out as a function of the aspect ratio $(L / D)$ of the particles, from the hard disc limit at one extreme $(L / D=0)$ to the thin hard needle limit at the other $(L / D=\infty)$. For long rods, a 2D nematic phase is observed at high density in which the orientational correlation functions decay algebraically, indicating that the phase does not possess true long range orientational order. The simulation data indicate that the transition from this phase to the low density isotropic phase is continuous, via a Kosterlitz-Thouless disclination unbinding type mechanism, rather than first order. For short rods the nematic phase disappears so that, on expansion, the solid phase undergoes a first order transition directly to an isotropic phase. Although the latter phase is globally isotropic, we find evidence for strong local positional and orientational correlations between the particles. Where possible, the simulation results are compared and contrasted to experimental, simulation and theoretical data for other two-dimensional liquid crystalline systems. (C) 2000 American Institute of Physics.
\end{abstract}

[S0021-9606(00)51016-2]

\section{INTRODUCTION}

Computer simulation studies of hard body particles have played an important role in the current understanding of the liquid crystalline behavior of systems composed of rodlike colloids. Following Onsager's theoretical demonstration that a system of infinitely long hard particles can exhibit an orientationally ordered phase if the density is sufficiently high, ${ }^{1}$ computer simulation studies gave the first indication that smectic phases can also be formed by systems of particles which interact through excluded volume interactions alone. ${ }^{2}$ Such discoveries have lead to further simulation and theoretical studies of simple hard body models and now the phase diagrams for monodisperse systems composed of, for example, ellipsoids ${ }^{3}$ and spherocylinders ${ }^{4}$ have been determined as a function of particle shape anisometry, ranging from their common limiting cases of hard spheres to infinitely thin rods. Other factors present in real colloidal systems which can influence the phase behavior, such as polydispersity in the length of the particles, have also been studied theoretically ${ }^{5}$ and via simulation. ${ }^{6}$ Although many model colloidal systems in three dimensions have been investigated and are now well understood, ${ }^{7}$ our understanding of two-dimensional (2D) liquid crystalline systems is far from complete.

Here we report a Monte Carlo simulation study of a family of 2D systems, composed of hard spherocylinders of aspect ratio $L / D$ confined to lie in a plane. Although these particles, defined as a rectangle of length $L$ and breadth $D$ capped at each end by a semicircle of diameter $D$, are more strictly described as "discorectangles," we shall use the term 2D spherocylinder throughout this paper. This model can be used to span an entire aspect ratio, from 2D hard disk ${ }^{8}$ at one extreme $(L / D=0)$ to $2 \mathrm{D}$ hard needles ${ }^{9}$ at the other $(L / D=\infty)$, and so we may expect to observe a variety of behavior as the aspect ratio of the particles is increased. However, we note that in 2D there is no distinction between prolate and oblate (rodlike and disklike) particles, as there is in $3 \mathrm{D}$, because particles in two dimensions have only two symmetry axes. Since we are particularly interested in the nature of any liquid crystalline phases present in the phase diagram, the relevant predictions about these phases are described in Sec. II. The simulations of systems composed of "long" and "short" rods confined to two dimensions are discussed in Secs. III and IV, respectively, and the distinction between them made clear. The phase diagram of $2 \mathrm{D}$ spherocylinders as a function of aspect ratio is presented in Sec. V, along with a comparison between this family of systems and other related models. Our conclusions are summarized in Sec. VI.

\section{2D NEMATIC PHASES}

One of the most interesting properties of $2 \mathrm{D}$ systems is the lack of true long range order (LRO). Although the correlation length in such a system may be macroscopically large, it is not infinite, as required for a true crystal. ${ }^{10}$ Straley has shown that, whilst true (orientational) LRO cannot exist for 2D nematics if the particles interact via a separable potential, this does not necessarily apply if the potential is not separable into positional and orientational parts. ${ }^{11}$ This decoupling of the potential is not possible for almost all models in which particle shape is taken into account including the present one. Thus it is important to know whether "realistic" liquid crystalline models that include a shape term (as opposed to a circular core) exhibit true LRO. To differentiate systems which possess true LRO from those that do not, those that lack true LRO are commonly referred to as having quasi-LRO. 
Quasi-LRO is to be expected in a 2D nematic if the free energy associated with collective fluctuations in the particle orientations is of the form

$$
F=\frac{1}{2} \int K(\nabla \theta(\mathbf{r}))^{2} d \mathbf{r}
$$

where $\theta(\mathbf{r})$ characterizes the orientation at position $\mathbf{r}$ with respect to a fixed axis and $K$ is the 2D Frank elastic constant. ${ }^{12}$ A more general form of Eq. (1) should contain two Franck constants $K_{\|}$and $K_{\perp}$ associated with distortions parallel and perpendicular to the localized director, but on a sufficiently large length scale these constants are renormalized to the same value $K .{ }^{12}$ It is useful to recall some predictions about the nature of a $2 \mathrm{D}$ nematic phase described by Eq. (1). The amplitude of the orientational fluctuations diverges logarithmically with the number of particles $N$,

$$
\left\langle\theta^{2}\right\rangle \sim \frac{k_{B} T}{4 \pi K} \ln N .
$$

This means that both the order parameter $S=\langle\cos (2 \theta)\rangle$ and the orientational correlation functions $g_{l}(r)=\langle\cos [l\{\theta(0)$ $-\theta(r)\}]\rangle(l=2,4, \ldots)$ decay algebraically and vanish in the limits $N \rightarrow \infty$ and $r \rightarrow \infty$, respectively,

$$
\begin{aligned}
& S \sim c N^{-k_{B} T / 2 \pi K}, \\
& g_{l}(r) \sim c^{\prime} r^{-\eta_{l}}=c^{\prime} r^{-l^{2} k_{B} T / 2 \pi K},
\end{aligned}
$$

where $c$ and $c^{\prime}$ are constants. One possible description of the transition between a 2D nematic phase with quasi-LRO and an isotropic phase is via a Kosterlitz-Thouless (KT) dislocation unbinding type mechanism. ${ }^{13}$ This transition is predicted to occur in 2D nematic systems at a critical value of the renormalized Franck constant $K_{c},{ }^{12}$

$$
\frac{\pi K_{c}}{8 k_{B} T}=1
$$

However, whilst this means that a nematic phase is not stable for values of the Franck constant below the critical value $K_{c}$, it does not rule out the possibility of a nematic-isotropic (NI) transition via a different mechanism at higher densities. The 2D version of the Onsager theory ${ }^{14}$ for infinitely thin rods confined to a plane predicts a continuous NI transition at a density $\rho L^{2} \approx 4.7$, although due to the neglect of third and higher virial coefficients this should be viewed with some caution. Simulations using systems of hard needles indicate that this model exhibits a continuous NI transition at $\rho L^{2} \approx 7,{ }^{9}$ via a KT disclination unbinding type mechanism, rather than a first order transition. Computer simulations have been performed on other nonseparable and separable potentials. Whilst quasi-LRO has been observed for the separable models studied, ${ }^{15,16}$ contrasting evidence is found for nonseparable potentials. ${ }^{9,16-18}$ Indeed, for ellipses, the nature of the transition appears to depend on the aspect ratio, and the phase diagram possesses a tricritical point at which the transition changes from first order to continuous. ${ }^{18}$

\section{PHASE BEHAVIOR OF LONG RODS IN 2D}

Monte Carlo simulations were used to study the phase behavior of 2D systems of spherocylinders of length $L / D=1,2,3,4,5,7,9$, and 15 . In this section we discuss the results of the long models, $L / D=9$ and 15 ; the shorter lengths exhibit a qualitatively different behavior and will be discussed in Sec. IV. The equation of state was determined using constant pressure (NPT) Monte Carlo simulations. ${ }^{19}$ For the two models $L / D=9$ and 15 , we performed simulations for systems of $640(80 \times 8)$ and $1200(150 \times 8)$ particles, respectively, using both expansion runs of a high density solid and compression runs of a low density gas. These sizes were chosen to ensure that the systems were essentially of equal dimension along the $x$ and $y$-axes. The length of the simulation at each state point varied, but was typically 50 000-100 000 cycles for equilibration and 100000 cycles for calculation of averages, although longer runs were used where this was deemed necessary, in the vicinity of the NI transition.

Since the behavior exhibited by both long rod systems is qualitatively similar, we discuss here only the results for $L / D=15$. The equation of state is shown in Fig. 1. At the highest pressure studied $\left(P=1.0\right.$, in units $\left.k_{B} T / D^{2}\right)$, the solid melts fairly rapidly to form a highly ordered nematic phase, in which the layered nature of the solid is completely lost. On both expansion of the high density nematic phase and compression of the low density isotropic phase, we do not find evidence for either hysteresis or a discontinuity in the equation of state and so it seems unlikely that there is a first order transition between the orientationally ordered and disordered states. However, the 2D nematic order parameter $S=\langle\cos (2 \theta)\rangle$ (see Table I), calculated via the usual diagonalization of the order parameter tensor, ${ }^{9}$ indicates that the orientational order of the system is dependent on density and, therefore, that there is a density driven transition from a disordered to an ordered state. To further investigate the nature of this transition, a number of constant volume runs were performed for a few different system sizes $(N=960,2500$, 6400 , see Table I) in the density range where the order parameter was observed to rapidly increase and we found that, as for $2 \mathrm{D}$ hard needles, ${ }^{9} \mathrm{~S}$ is strongly dependent not only on density, but also on the system size. This strong system size dependence is exactly what we expect for a system with quasi-LRO [see Eq. (3)]. Indeed, if the orientational correlation function in the nematic phase decays algebraically, we expect that the nematic order parameter $S$ will tend to zero for all densities as the system size is increased towards the thermodynamic limit. For a finite system, we also expect that the order parameter of the isotropic phase will be larger than zero once the correlation length of nematic fluctuations exceeds the size of the periodic simulation box. Whilst this means that the order parameter and the equation of state alone do not help us to determine the location of the transition between the isotropic and nematic phases, analysis of the system size dependence of the order parameter is useful. Since the nematic order parameter is expected to decay as $S \sim N^{-k_{B} T / 2 \pi K}$ [Eq. (3)], we can use the system size dependence to obtain the Franck constant $K$ as a function of density. This in turn allows us to determine the location of the 

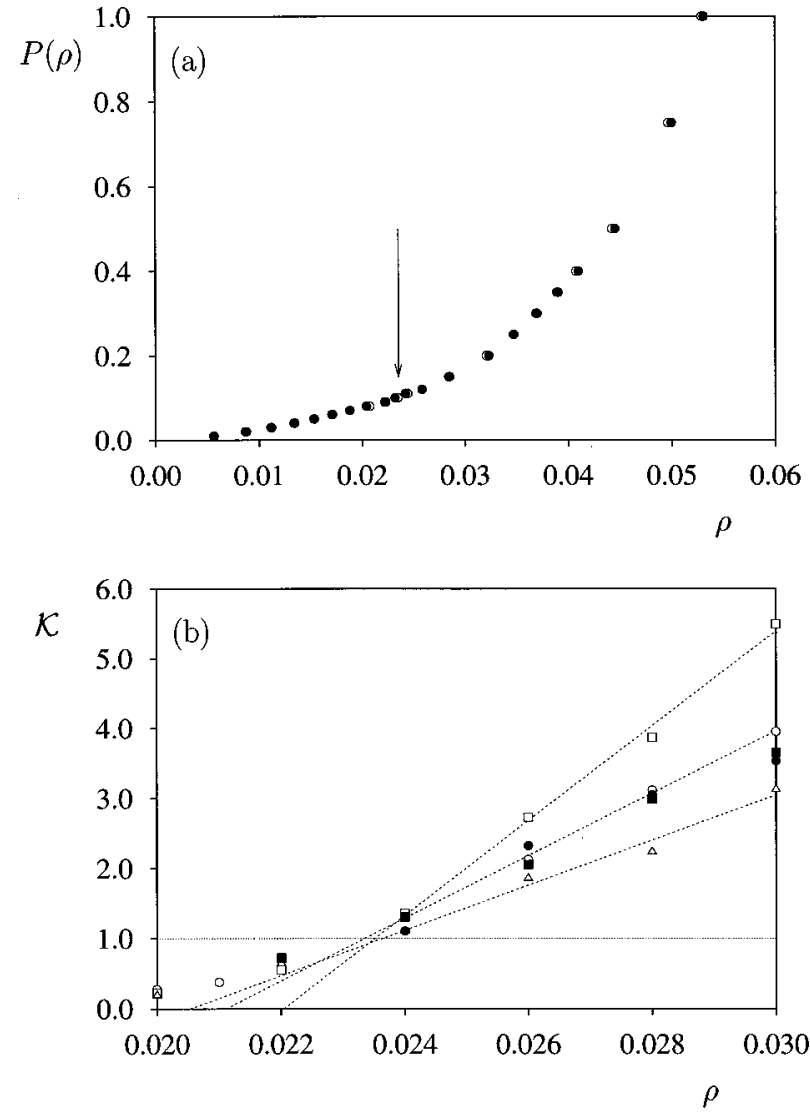

FIG. 1. (a) The equation of state for a 2D system of 1200 spherocylinders of aspect ratio $L / D=15$ determined from NPT simulations; (O) compression from isotropic, $(\bullet)$ expansion from perfectly ordered nematic. The arrow indicates the location of the Kosterlitz-Thouless disclination unbinding type transition between the isotropic and nematic phases. (b) The renormalized Franck constant $\mathcal{K}=\pi K / 8 k_{B} T$ for spherocylinders of length $L / D=15$ determined from NVT simulations by block averaging the nematic order parameter for systems of $(\square) 960,(\bigcirc) 2500$, and $(\triangle) 6400$ particles and from the algebraic decay of the orientational correlation function $g_{l}(r)$ [see Eqs. (3) and (4)] for $(-) l=2$ and (ם) $l=4$ for the larger system. Units, $P$ in $k_{B} T / D^{2}, \rho$ in $D^{-2}$.

disclination unbinding transition, below which there is no possibility of a stable nematic phase.

The block averaging analysis of the order parameter, which we briefly describe, follows that previously used for the systems of infinitely thin rods. ${ }^{9}$ For each of the constant volume runs, the values of $S, S^{2}$, and $S^{4}$ were calculated for the whole system $(N=960,2500$ or 6400$)$ and subsystems with an area equal to $1 / 4,1 / 16,1 / 36,1 / 64$ and, for the largest system, $1 / 100$ of the simulation box for densities in the range $0.020<\rho<0.030$ (units $D^{-2}$ ). The (sub)system size dependence of the order parameter $S$ within the three different systems was then fitted to a power law of the form $S=a N^{-b}$ [see also Eq. (3)], with the coefficient $b$ related to the Franck constant by $b=k_{B} T / 2 \pi K$. Similarly the size dependencies of $S^{2}$ and $S^{4}$ gave constants $b_{2}$ and $b_{4}$, related to $b$ by $b_{2}=2 b$ and $b_{4}=4 b$. For densities $\rho \geqslant 0.022$, we find that the relation $b=b_{2} / 2=b_{4} / 4$ is well satisfied as expected in the nematic phase. In Fig. 1(b), the elastic constant $\mathcal{K}=\pi K / 8 k_{B} T=1 / 16\langle b\rangle$ is plotted as a function of density, where $\langle b\rangle$ is the average of $b, b_{2} / 2$, and $b_{4} / 4$. We recall that spontaneous disclination unbinding is expected if
TABLE I. Summary of the thermodynamic properties obtained from Monte Carlo simulations of a system of $2 \mathrm{D} L=15$ spherocylinders. Column 1: simulation ensemble, NPT or NVT; column 2: number of particles; column 3: pressure, in units $k_{B} T / D^{2}$; column 4: density, in units $D^{-2}$; column 5: 2D orientational order parameter $S=\langle\cos (2 \theta)\rangle$.

\begin{tabular}{crccc}
\hline \hline Ensemble & \multicolumn{1}{c}{$N$} & $P$ & $\rho$ & $S$ \\
\hline NPT & 1200 & 0.02 & 0.00864 & 0.0295 \\
NPT & 1200 & 0.04 & 0.01345 & 0.0499 \\
NPT & 1200 & 0.06 & 0.01710 & 0.0904 \\
NPT & 1200 & 0.08 & 0.02072 & 0.1034 \\
NPT & 1200 & 0.09 & 0.02216 & 0.3595 \\
NPT & 1200 & 0.10 & 0.02345 & 0.5377 \\
NPT & 1200 & 0.15 & 0.02837 & 0.7822 \\
NPT & 1200 & 0.20 & 0.03207 & 0.8749 \\
NPT & 1200 & 0.25 & 0.03473 & 0.8812 \\
NVT & 960 & $\ldots$ & 0.020 & 0.183 \\
NVT & 2500 & $\ldots$ & 0.020 & 0.144 \\
NVT & 6400 & $\ldots$ & 0.020 & 0.095 \\
NVT & 960 & $\ldots$ & 0.022 & 0.504 \\
NVT & 2500 & $\ldots$ & 0.022 & 0.453 \\
NVT & 6400 & $\ldots$ & 0.022 & 0.378 \\
NVT & 960 & $\ldots$ & 0.024 & 0.714 \\
NVT & 2500 & $\ldots$ & 0.024 & 0.702 \\
NVT & 6400 & $\ldots$ & 0.024 & 0.694 \\
NVT & 960 & $\ldots$ & 0.026 & 0.813 \\
NVT & 2500 & $\ldots$ & 0.026 & 0.777 \\
NVT & 6400 & $\ldots$ & 0.026 & 0.752 \\
NVT & 960 & $\ldots$ & 0.028 & 0.830 \\
NVT & 2500 & $\ldots$ & 0.028 & 0.810 \\
NVT & 6400 & $\ldots$ & 0.028 & 0.792 \\
\hline \hline
\end{tabular}

$\pi K / k_{B} T<8$, that is, if $\mathcal{K}<1$. The simulation data for all three system sizes indicates that the nematic is no longer stable below a density $\rho \approx 0.0235$. We also observe that the elastic constant evaluated at constant density is dependent on the system size; this has also been observed for other systems. ${ }^{9}$ However, whilst increasing the system size appears to renormalize the effective Franck constant to lower values, this does not appear to shift the transition to higher densities, at least within the computational accuracy for the system sizes and the model studied here [see Fig. 1(b)].

Evidence that the orientational correlations in the nematic phase decay algebraically comes from the angular correlation functions $g_{l}(r)$, shown in Fig. 2(a) for the lowest density at which the $2 \mathrm{D}$ nematic phase is stable $[\rho=0.024$, see also Fig. 2(b)]. Since these are expected to decay as $g_{l}(r) \propto r^{-} \eta_{l}$, with $\eta_{l}=l^{2} k_{B} T / 2 \pi K$ [see Eq. (4)] at distances longer than the particle length, analysis of these functions can also give information on the location of the disclination unbinding transition via the density dependence of the Franck constant. The elastic constants obtained from the correlation functions $g_{2}(r)$ and $g_{4}(r)$ for the largest system are plotted in Fig. 1(b). Although the values are slightly higher than those obtained from the block averaging of the order parameter for the same system, they do confirm the location of the continuous transition at $\rho \approx 0.0235$. Note that the orientational correlation functions $g_{l}(r)$ also show some system size dependence [see Fig. 2(a)]. For example, the correlation function $g_{2}(r)$ determined for the smaller system $(N=960)$ in the range $r<5 L$ decays algebraically but slower than that determined for the larger system $(N=6400)$. This, of course, 
(a)

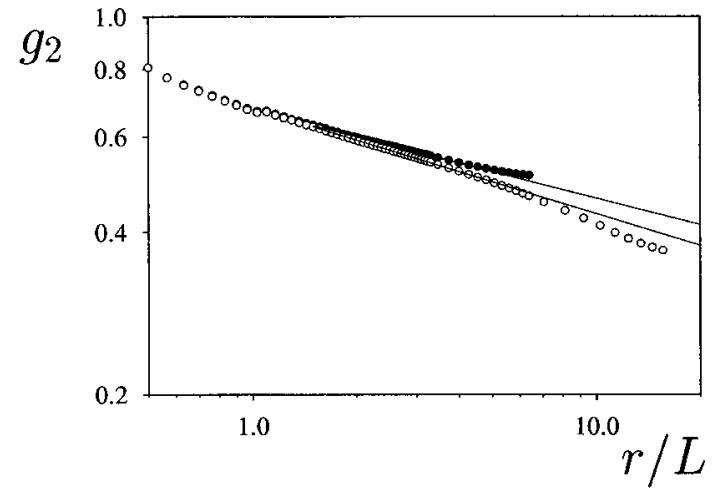

(b)

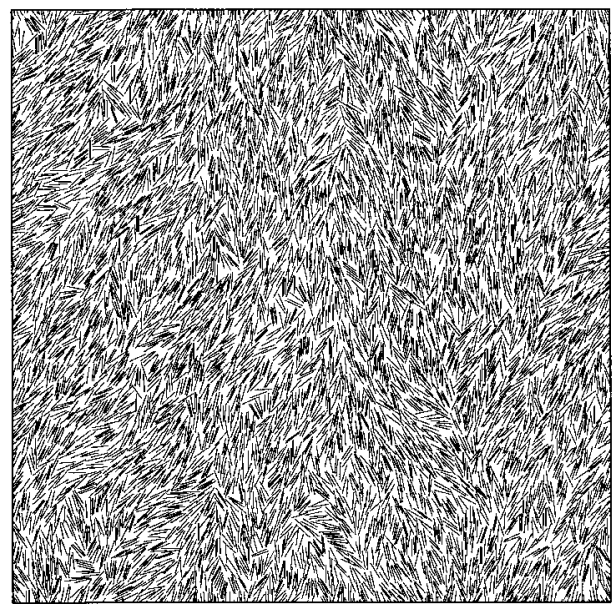

FIG. 2. (a) The orientational correlation function $g_{2}(r)=\langle\cos [2\{\theta(0)$ $-\theta(r)\}]\rangle$ for systems of $(\bullet) 960$ and $(\bigcirc) 6400$ spherocylinders of length $L / D=15$ at a density $\rho=0.024$, just above the Kosterlitz-Thouless transition. The solid lines indicate the algebraic decay $\left(g_{2}(r) \propto r^{-} \eta_{2}\right)$ of the orientational correlation function, fitted over the range $1.5<r / L<5$.0. (b) $\mathrm{A}$ typical snapshot of a configuration of $64002 \mathrm{D}$ hard spherocylinders at this density. The spherocylinders are shown as thin lines for clarity.

would lead to a larger effective Franck constant for the smaller system, which is exactly what we observe in Fig. 1(b) from the subsystem size analysis. This is most probably due to the periodic nature of the boundary conditions, which tend to enhance the orientational order for the small system. For larger distances the decay appears to be slower, although since these separations are approaching half the simulation box dimension, we may expect to observe enhanced correlations with the periodic images. For the larger system the decay for separations $r>6 L$ is slightly faster than the algebraic decay observed over the range $1.5 L<r<5 L$. However, we did not use the entire range to obtain estimates of the Franck constant because of the slow equilibration of long wavelength fluctuations of such large systems. Allowing for this faster decay when determining $\eta_{l}$ from the correlation function would slightly lower the effective elastic constant and this is presumably why the subsystem block analysis, which does take the whole system into account, gives a marginally lower value [see Fig. 1(b)]. A typical snapshot of this system in the vicinity of the transition is shown in Fig. 2(b).

We end this section by concluding that the behavior of 2D spherocylinder systems for aspect ratios $L / D=9$ and above is similar to that observed for infinitely thin needles. ${ }^{9}$

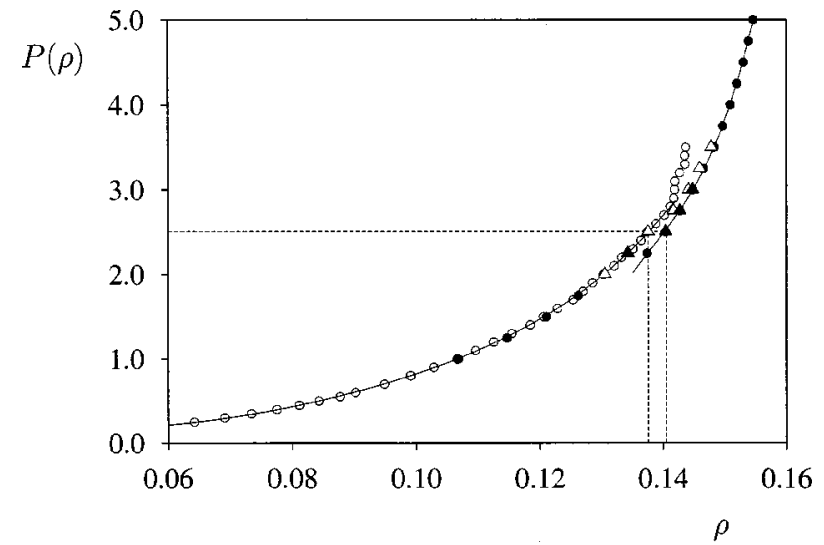

FIG. 3. Equation of state for a 2D system of 840 spherocylinders of aspect ratio $L / D=5$ determined from NPT simulations; $(\bigcirc)$ compression from isotropic, $(\bullet)$ expansion from solid and $(\triangle)$ compression and $(\boldsymbol{\Delta})$ expansion using square-flip trials. The dotted lines indicate the coexistence pressure and the coexistence densities of the isotropic and solid phases obtained from free energy calculations. Units, $P$ in $k_{B} T / D^{2}, \rho$ in $D^{-2}$.

Thus a 2D nematic phase, in which the orientational correlations decay algebraically, is observed. This phase undergoes a continuous transition to an isotropic phase, via a Kosterlitz-Thouless disclination unbinding type mechanism, which can be located by determining the density dependence the effective Franck constant. Below this critical density the nematic phase is no longer stable, although the finite order parameter observed for small systems indicates that strong local nematic fluctuations persist over a narrow density range below the transition. In contrast to the $2 \mathrm{D}$ needle system which, by definition, is at zero volume fraction, we expect to observe a solid phase at high densities (finite volume fractions) for finite aspect ratios. However, for the systems studied, the nematic phase appears to be stable up to rather high densities. At higher densities, relaxation of the system is too slow to be able to investigate the melting behavior accurately.

\section{PHASE BEHAVIOR OF SHORT RODS IN 2D}

The behavior exhibited by shorter rods is found to be rather different from that just described for longer rods. Constant pressure simulations were performed for aspect ratios $L / D=1,2,3,4,5$, and 7 , for systems composed of between 600 and 1000 particles using compression and expansion runs, as before. A typical equation of state is shown in Fig. 3 for spherocylinders of aspect ratio $L / D=5$; since all the shorter models exhibit the same qualitative behavior, we shall discuss the results for this system only. The first major difference between short and long rods can be observed directly from the equation of state. The strong hysteresis and offset between the branches of the equation of state are clearly indicative of a first order transition. On expansion, the order parameter $S$ (not shown) is found to drop from almost unity to essentially zero when the system jumps from one branch to the other. From this we can almost certainly conclude that the short rod systems do not exhibit a nematic phase, but undergo a transition directly from the solid to an isotropic phase. To determine the location of the transition, 
we followed the standard route of calculating the Gibbs free energy of each phase as a function of pressure. ${ }^{20}$ For the isotropic phase, we can compute the absolute value of the free energy via a thermodynamic integration along the equation of state taking the ideal gas as a reference state. For the solid phase, we choose as a reference system an Einstein crystal, with a Hamiltonian which couples the particles to their equilibrium lattice positions and the orientations to an aligning field; this Hamiltonian takes the form ${ }^{4}$

$$
H_{\lambda_{1}, \lambda_{2}}=\lambda_{1} \sum_{i}\left(\mathbf{r}_{i}-\mathbf{r}_{i}^{0}\right)^{2}+\lambda_{2} \sum_{i} \sin ^{2} \theta_{i}
$$

where $\mathbf{r}_{i}$ and $\mathbf{r}_{i}^{0}$ are the position and lattice site of particle $i$, $\theta_{i}$ is the angle between the particle and aligning field and $\lambda_{1}$ and $\lambda_{2}$ are the coupling constants. Further details of the calculation of the free energy of the solid phase of a system with orientational and positional order can be found in Ref. 4 , with minor corrections for the fixed center-of-mass constraint in Ref. 21. The coexistence pressure and densities determined for the $L / D=5$ system are shown in Fig. 3 .

Further evidence that nematic phases are not observed for the short rod models comes from the observation that the systems were unable to jump from the low density branch of the equation of state to the high density branch. We recall that for a long rod system, the correlation length of nematic fluctuations gradually increases with density within the isotropic phase. As the density is further increased the system becomes nematic and the orientational order continues to steadily increase whilst the system remains fluid. Thus we do not expect the orientational degrees of freedom to play a significant role in the freezing transition, as the particles are all essentially aligned prior to the transition to the solid (even though this occurs at too high a pressure to be characterized in our simulations). However, for the short rod systems, the lack of orientational order prior to the freezing transition leads to the system being unable to jump to the solid branch of the equation of state, even for extremely long simulation runs of over a million cycles (for the $L / D=5$ system). One reason for this can be observed in snapshots of the two phases either side of the solid-isotropic transition; typical examples of these are shown in Figs. 4(a) and 4(b) for an expansion run of a system of 840 particles. We observe that the high density phase is highly ordered, both orientationally and positionally, although we have not investigated whether this phase should strictly be called a 2D solid or a 2D smectic. On melting [see Fig. 4(b)] the system clearly becomes isotropic. However, the isotropic phase does not contain domains reminiscent of a nematic phase (which are observed in the isotropic phase of the long rod systems), but rather chains of particles aligning side-by-side, more reminiscent of the solid. A similar structure is observed for all short rod systems, even $L / D=1$, on both expansion of the solid and compression of the fluid, although the chains appear to become less well developed as the particle length decreases. The equation of state (see Fig. 3) indicates that on further compression of the isotropic phase, the solid phase is not recovered. This seems entirely reasonable when we consider that locally, the isotropic phase is essentially locked into a solid
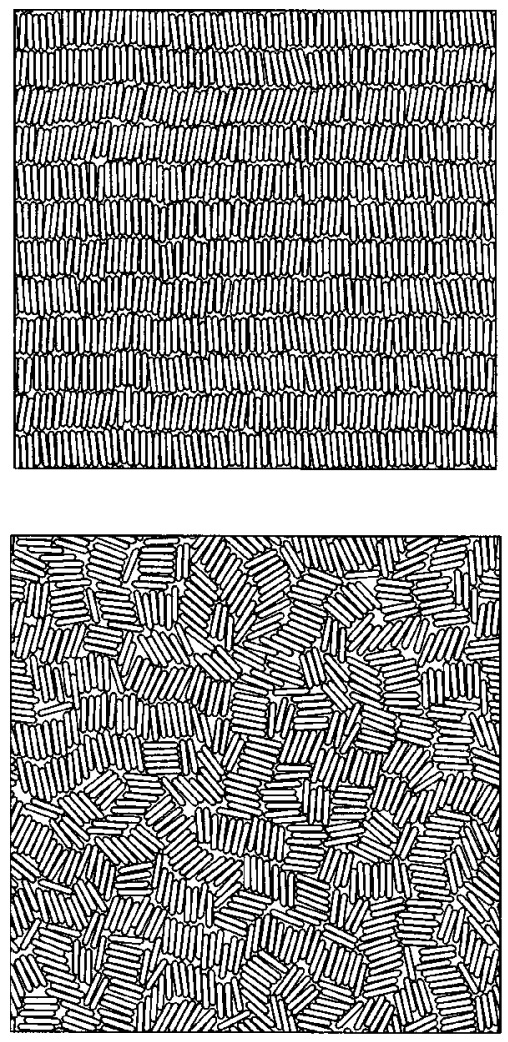

(c)

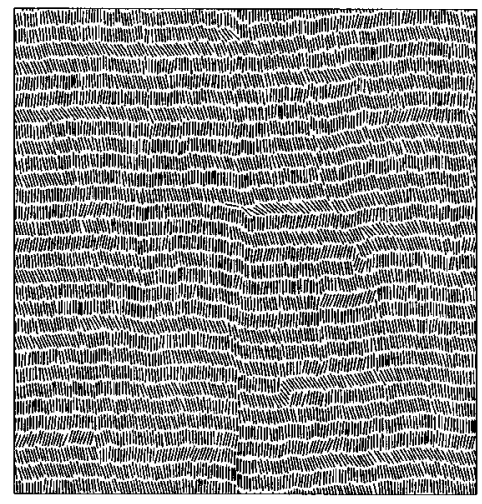

(d)

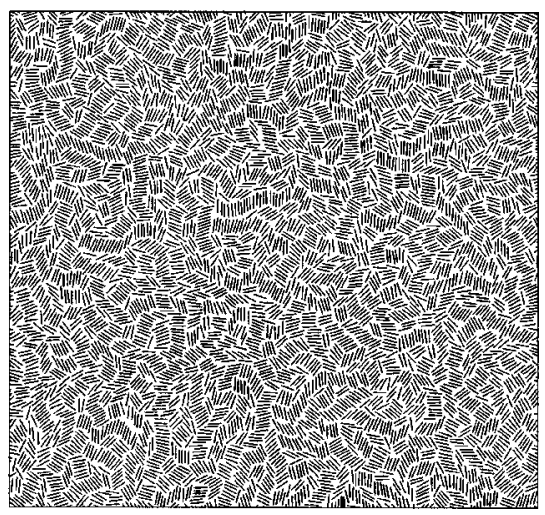

FIG. 4. Snapshots of systems of spherocylinders of length $L / D=5$ in the vicinity of the melting transition taken from NPT simulations. (a) The solid phase at $P=3.0, \rho \approx 0.145$ and (b) the isotropic phase at $P=0.2, \rho \approx 0.130$ of a system of 840 particles. Snapshots of the melting of a solid of 2400 particles at (c) $P=2.25$ and (d) $P=2.00$. Units, $P$ in $k_{B} T / D^{2}, \rho$ in $D^{-2}$. 
like structure. Conventional single particle trial moves used in the Monte Carlo simulations do not help to unlock this bottleneck; large angular displacement trials $\left(90^{\circ}\right)$ are always rejected, whilst small successful displacements do not result in any significant changes. To overcome this problem, we performed trials in which small regions containing more than one particle were rotated; thus in addition to single particle trials, which help the system relax locally, trials were performed in which a square region, of dimensions approximately equal to the length of a single particle, was rotated by $\pm 90^{\circ}$. Although most of these were rejected due to overlap, occasional successes $(\approx 0.1 \%)$ in the vicinity of the melting transition $(0.135 \leqslant \rho \leqslant 0.145)$ do indeed lead to significant changes in structure. The data obtained from the use of these trials in a compression run are shown in Fig. 3. The "squareflip" trials are found to reproduce the jump in the equation of state on compression (after $100000 \mathrm{~s}$ of cycles), although the densities are slightly lower than those obtained on expansion. Snapshots (not shown) indicate that this is because the system is composed of two large solid domains, perpendicularly aligned, rather than a single domain. Given even longer runs the system may gradually transform into a single domain, but these were not performed. The square-flip trial moves also helped to rule out another possibility. The equation of state indicates that the solid can be overexpanded past the melting density determined from the free energy calculations. In large systems this leads to a highly defected layered structure [see Fig. 4(c)] which appears to be stable over hundreds of thousands of cycles. Lowering the pressure slightly leads to the layer structure being destroyed, in favor of the isotropic phase composed of chains [see Fig. 4(d)]. However, when the same expansion run is performed using square-flip trials, the overexpanded metastable layered system is found to disorder. Thus we conclude that this highly defected structure is not thermodynamically stable. The fact that the coexistence pressure determined from the free energy calculations lies at the lower end of the hysteresis region obtained using the square-flip Monte Carlo trials, which can equilibrate the system much more efficiently than standard trials, gives us increased confidence in the accuracy of the free energy calculations.

The snapshots of the isotropic phase of the $L / D=5$ system indicate that not only are chains formed, but that neighboring chains appear to align either parallel or perpendicular to each other. This may lead us to wonder if this phase could exhibit a tetratic phase, the equivalent of the cubatic phase in three dimensions, ${ }^{22,23}$ in which there are two preferred directions of alignment, but no positional order. However, this can be ruled out by analyzing the orientational correlation functions. The second and fourth rank correlation functions, resolved into directions parallel and perpendicular to the particle at the origin, are shown in Fig. 5. These indicate the high local orientational and positional order in the isotropic phase; we recall that parallel alignment contributes positively to both $g_{2}$ and $g_{4}$, whereas perpendicular alignment contributes negatively to $g_{2}$ but positively to $g_{4}$. The peaks along the $r_{\perp}$ direction clearly arise from parallel particles within the same chain. The structure along the $r_{\|}$axis is less well defined. $g_{2}\left(r_{\|}, 0\right)$ has a strong minimum between
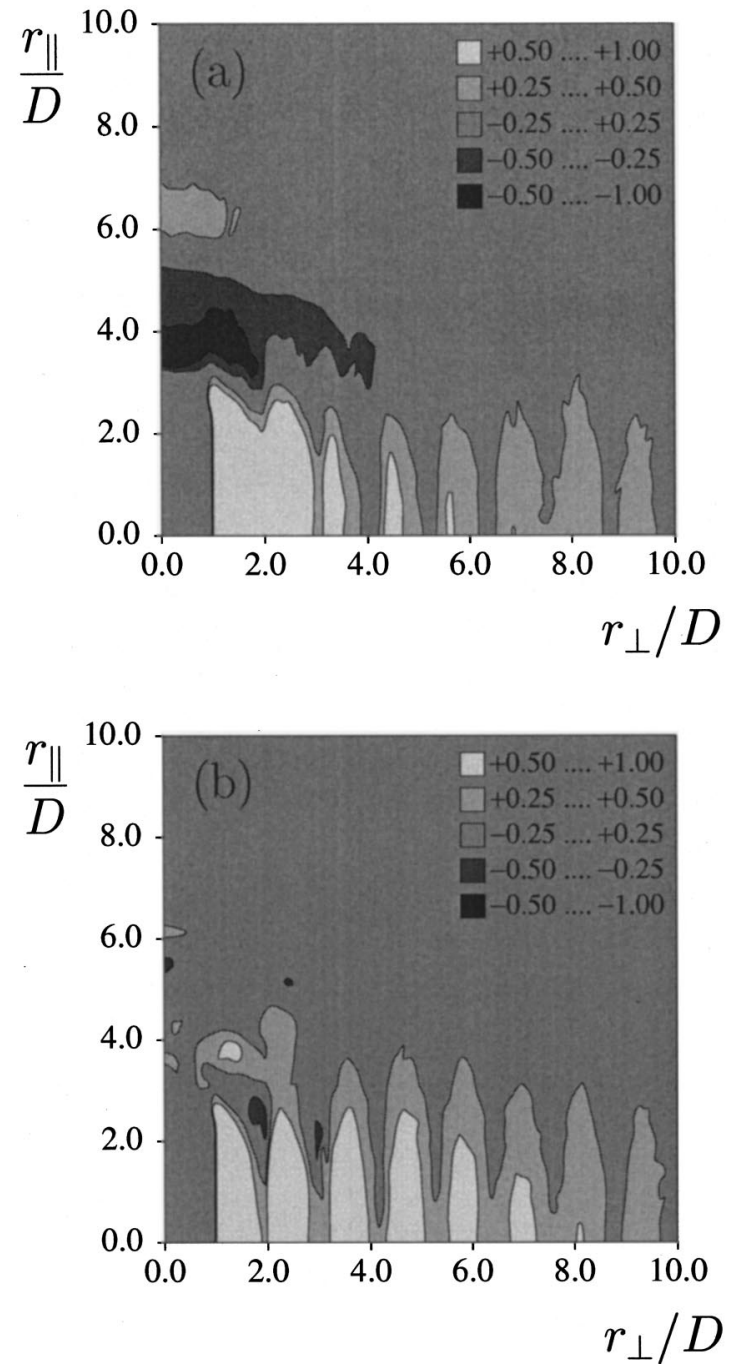

FIG. 5. The orientational correlation functions (a) $g_{2}(\mathbf{r})=\langle\cos [2\{\theta(\mathbf{0})$ $-\theta(\mathbf{r})\}]\rangle$ and (b) $g_{4}(\mathbf{r})=\langle\cos [4\{\theta(\mathbf{0})-\theta(\mathbf{r})\}]\rangle$ for an isotropic system of 1000 particles of aspect ratio $L / D=5$ at a density $\rho=0.125$. The interparticle vector $\mathbf{r}$ is resolved into components $r_{\|}$and $r_{\perp}$, parallel and perpendicular to the orientation of the particle at the origin.

$3.0 D<r_{\|}<4.0 D$ which occurs for steric reasons; for two particles to approach this close, they almost certainly will be perpendicular to each other. A secondary maximum at $r_{\|}$ $\approx 6.5 \mathrm{D}$ can be identified as arising from two parallel particles although this is rather weak and does not extend along the $r_{\perp}$ direction, indicating that the chains do not stack on top of each other (as they do in the solid phase). If the structure was tetratic, then we expect this to show up in the long range region of $g_{4}(r)$ which should be finite, in contrast to $g_{2}(r)$ which will tend to zero. However, the observation that $g_{4}\left(r_{\|}, r_{\perp}\right)$ decays to zero rather quickly away from the $r_{\perp}$ axis indicates that the phase does not possess tetratic order.

\section{PHASE DIAGRAM OF 2D SPHEROCYLINDERS}

The results of the NPT simulations and free energy calculations to determine the coexistence densities of the solidisotropic transition exhibited by the short rod systems are summarized in Fig. 6. This figure indicates that the volume fraction at which the solid melts is essentially constant and 

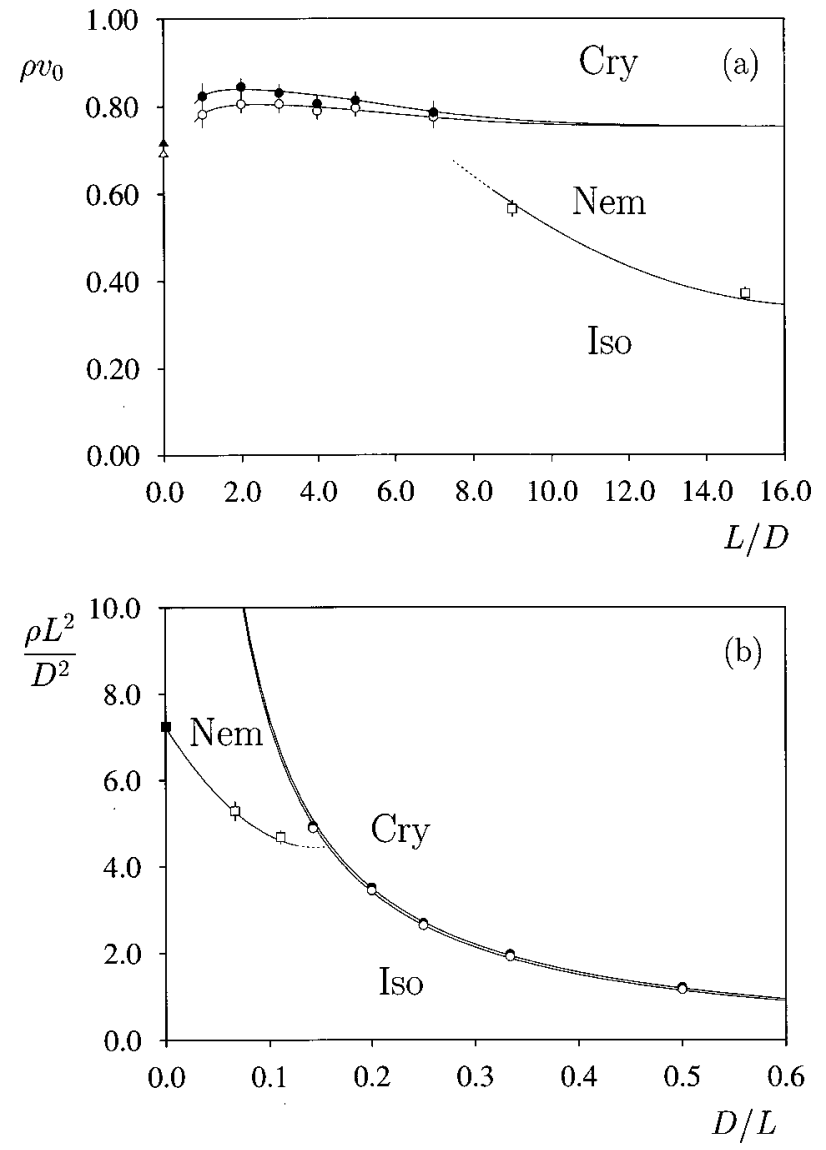

FIG. 6. Phase behavior of 2D systems of spherocylinders as a function of their aspect ratio $L / D$. $(\bigcirc, \bullet)$ Solid-isotropic transition, $(\square)$ 2D nematicisotropic transition, $(\triangle, \boldsymbol{\Lambda})$ melting transition in 2D disks (Ref. 8) and (ם) NI transition in 2D needles (Ref. 9). The lines are a guide to the eye only.

not dependent on the length of the rod, although the density gap between the two phases is reduced as the length is increased. We note that there is still controversy surrounding the melting transition for the limiting case $L \rightarrow 0$, that is, 2D hard disks. For this model, it is not clear whether the melting transition is first order, continuous or of the KosterlitzThouless-type. This point is further addressed in Refs. 24 and 25. However, whilst the order of the transition is not clear, the approximate density region in which the transition is located is not in doubt. The data in Fig. 6 indicate that the volume fraction at melting for elongated particles is larger than that for $2 \mathrm{D}$ disks. This is because the orientational degrees of freedom present in the rod systems help to destroy the solid phase in favor of the isotropic phase. We have found no evidence for topological defects occurring even for the shortest rods $L=1$. However, similar to the situation for 2D hard disks, we cannot totally rule out the possibility that a Kosterlitz-Thouless-type melting transition occurs for very short rods. The melting transition for lengths above $L / D=7$ has not been studied, due to the long equilibration times necessary to accurately locate the transition. However, it would be interesting to determine the volume fraction of the limiting case of infinitely thin rods at the melting transition; although this transition is expected to occur at infinite number density, it will occur at finite volume fraction. By assuming a constant director for the nematic phase over a simulation cell, a simple scaling along the director will transform a system of infinitely long rods into a system of particles with finite volume and so the melting transition could be studied in the finite length limit. This has been done for $3 \mathrm{D}$ systems of rods ${ }^{4}$ and disks ${ }^{26}$ but not yet for $2 \mathrm{D}$ systems. The data are plotted to show the behavior in the limit $L / D$ $\rightarrow \infty$ in Fig. 6(b). The simulation data for $L / D=9$ and 15 clearly indicate that there is a drop in the concentration $\left(\rho L^{2} / D^{2}\right)$ at which the KT disclination unbinding transition takes place as the rod length is shorter, as we expect since the width of the particles becomes more and more important as the rod becomes shorter. The nematic-isotropic transition disappears in the region $6<L / D<9$. We did not observe a NI transition in the simulations for $L / D=7$, although since only relatively small systems were used we cannot rule this out. In the simulations, the order parameter in the fluid at the melting transition was reasonably high $(S \approx 0.20-0.40)$ and subject to large fluctuations, which could possibly mean that a nematic is stable for this aspect ratio, although no system size dependence was studied. The square-flip moves which were used for the $L / D=5$ system were found to be less useful as the rod length increases, since the square necessary to flip a region of dimension $(L / D)^{2}$ contains more particles and so is more likely to be rejected. Snapshots from the simulations indicate that there are some chains of particles similar to those formed in the isotropic phase of the $L / D$ $=5$ model, although these tend to be more disordered within the chain, and also that there are also some regions similar to those of the nematic of the $L / D=9$ model. Clearly larger systems would be necessary to determine whether or not there is a stable nematic phase at $L / D=7$. Figure 6(b) indicates that even though this is a strong possibility, the density range of the nematic would be severly reduced in comparison to the longer rods $L / D=9$ and 15 , and so determining the location of the KT transition may be difficult.

In summary, we observe two types of behavior for spherocylinders confined to a plane. Long rods $(L / D>7)$ have a behavior similar to infinitely thin needles, in that the exhibit a 2D nematic phase with algebraic decay in the orientational correlations between the particles. Shorter rods $(L / D<7)$ do not exhibit a nematic phase, but undergo a melting transition to a phase dominated by chains of particles which align side-by-side, but are isotropically arranged. Thus we observe that for short rods, the most favorable packing to maximize the entropy on melting is to retain the local solidlike order and form an isotropic distribution of chains of particles. A crossover in behavior occurs at $L / D \approx 7$ when it is more favorable to lose the local positional order between particles but to retain the orientational order and so form a nematic phase. We conclude that the enhanced stability of the isotropic phase due to the formation of chains means that the nematic phase does not enter the phase diagram until $L / D \approx 7$; this contrasts to the $3 \mathrm{D}$ case, where the nematic phase is observed for rods as short as $L / D=4$. $^{4}$

It is interesting to compare the results of these simulations with other experiments and models of 2D liquid crystalline systems. Studies of 2D hard ellipses ${ }^{18}$ indicate that the nematic is stable for aspect ratios of $k \approx 4$ and above; we should compare the behavior of ellipses of aspect ratio $k$ with 
spherocylinders of length $(L+D) / D$. However, the NI transition appears to be continuous for $k=6$ but first order for $k=4$, and thus a tricritical point is expected between these two aspect ratios. The fact that short ellipses do not exhibit a similar isotropic phase to spherocylinders is not surprising, since spherocylinders can pack most effectively side-by-side. In contrast, ellipses aligned side-by-side can roll against each other and therefore it is unlikely that chains will form and so the isotropic phase observed for spherocylinders is not as favorable as a nematic phase. This also appears to be the case for the high density solid phase. For the ellipses, rather large fluctuations in the positions of the layers are observed. In contrast, the spherocylinders tend to exhibit almost perfected ordered layers due to the effective side-by-side alignment. Thus we observe that a small difference in the shape of the particle can lead to rather different phase behavior. Scaled particle theory for 2D systems of ellipses and rectangles have also been performed. ${ }^{27}$ Whilst this theory does not take into account either the solid phase or the possibility to form chains in the isotropic phase, we can compare the results for the critical aspect ratio for the nematic phase to be observed with that determined for rectangles (the same as our model except with no end-caps). We recall that for spherocylinders, a nematic phase is observed only for $L / D \approx 7$ or larger and that the NI transition is always continuous. Scaled particle theory predicts that a continuous NI transition occurs if $L / D>5.44$ and a first order NI transition occurs if $L / D<5.44$. If the semicircular end caps on the spherocylinders behave as extra square units of length $D / 2$, the tricritical point would occur for spherocylinders at $L / D=4.44$, although somewhere between $L / D=4.44$ and 5.44 is more likely. Thus it seems reasonable that the NI transition for spherocylinders is always continuous, since we find that the nematic phase is only observed at densities well above this predicted nematic-isotropic tricritical point. However, it would be interesting to compare the results with a theory that does take into account the possibility of the particles aligning side-by-side in chains, since this seems to be the reason for the enhanced stability of the isotropic phase over the nematic for short rods. The only comparable experimental system is that of long phospholipid tubules suspended on a surface in which the NI transition is claimed to be first order. ${ }^{28}$ However, it seems likely that this is due to aggregration of particles in the dense nematic phase, since this phase coexists with an isotropic phase of very low density.

\section{CONCLUSIONS}

We have studied the phase behavior of a family of $2 \mathrm{D}$ systems composed of rigid rods confined to lie in a plane, as a function of the rod length. Systems composed of particles longer than $L / D \approx 7$ are found to undergo a transition from the isotropic fluid to a 2D nematic phase. The orientational correlations in this phase decay algebraically and thus suggest that this phase does not possess true long range order. Since this is also the case for the limiting case of infinitely thin needles, this further suggests that the nematic phase for all aspect ratios $L / D$ (where observed) will have the same behavior. For each model system exhibiting a nematic phase, we have performed a block averaging analysis of the order parameter to obtain an estimate of the renormalized Franck elastic constant $K$, to determine the location of the Kosterlitz-Thouless disclination unbinding transition. The solid-isotropic transition density drops below the extrapolated KT transition density at $L / D \approx 7$ and so for shorter rods we no longer observe a stable $2 \mathrm{D}$ nematic phase. Although the low density phase is isotropic, at densities below melting we still observe a strong local correlation in the positions and orientations of the particles. These correlations lead to the formation of chains of rods, in which the particles tend to align side-by-side. Examination of the orientational correlation functions $g_{l}\left(r_{\|}, r_{\perp}\right)$ appears to rule out the chance that this short range order can lead to the formation of a $2 \mathrm{D}$ tetratic phase in these systems.

\section{ACKNOWLEDGMENTS}

The work of the FOM Institute is part of the research program of FOM (Stichting Fundamenteel Onderzoek der Materie) and is supported by NWO (Nederlandse Organisatie voor Wetenschappelijk Onderzoek). M.A.B. acknowledges the financial support of the EU through the Marie Curie TMR Fellowship Programme. We are grateful to M. Noro and B. Groh for careful readings of the manuscript.

${ }^{1}$ L. Onsager, Ann. (N.Y.) Acad. Sci. 51, 627 (1949).

${ }^{2}$ D. Frenkel, H. N. W. Lekkerkerker, and A. Stroobants, Nature (London) 332, 822 (1988).

${ }^{3}$ D. Frenkel and B. M. Mulder, Mol. Phys. 55, 1171 (1985).

${ }^{4}$ P. G. Bolhuis and D. Frenkel, J. Chem. Phys. 106, 666 (1997).

${ }^{5}$ T. J. Sluckin, Liq. Cryst. 6, 111 (1989).

${ }^{6}$ M. A. Bates and D. Frenkel, J. Chem. Phys. 109, 6193 (1998).

${ }^{7}$ M. P. Allen, G. T. Evans, D. Frenkel, and B. M. Mulder, Adv. Chem. Phys. 86, 1 (1993).

${ }^{8}$ B. J. Alder and T. E. Wainwright, Phys. Rev. 127, 359 (1962).

${ }^{9}$ D. Frenkel and R. Eppenga, Phys. Rev. A 31, 1776 (1985).

${ }^{10}$ K. Huang, Statistical Mechanics (Wiley, New York, 1963).

${ }^{11}$ P. Straley, Phys. Rev. A 4, 675 (1971).

${ }^{12}$ D. R. Nelson and R. A. Pelcovits, Phys. Rev. B 16, 2191 (1977).

${ }^{13}$ J. M. Kosterlitz and D. Thouless, J. Phys. C 6, 1181 (1973).

${ }^{14}$ R. F. Kayser and H. J. Raveché, Phys. Rev. A 17, 2067 (1978).

${ }^{15}$ J. Tobochnik and G. V. Chester, Phys. Rev. B 20, 3761 (1979).

${ }^{16}$ J. Tobochnik and G. V. Chester, Phys. Rev. A 27, 1221 (1983).

${ }^{17}$ J. Vieillard-Baron, J. Chem. Phys. 56, 4729 (1972).

${ }^{18}$ J. A. Cuesta and D. Frenkel, Phys. Rev. A 42, 2126 (1990).

${ }^{19}$ M. P. Allen and D. J. Tildesley, Computer Simulation of Liquids (Clarendon, Oxford, 1987).

${ }^{20}$ D. Frenkel and A. J. C. Ladd, J. Chem. Phys. 81, 3188 (1984).

${ }^{21}$ J. M. Polson, E. Trizac, S. Pronk, and D. Frenkel, J. Chem. Phys. (submitted).

${ }^{22}$ D. Frenkel, in Liquids, Freezing, and the Glass Transition, edited by J. P. Hansen, D. Levesque, and J. Zinn-Justin (North-Holland, Amsterdam, 1991).

${ }^{23}$ J. A. C. Veerman and D. Frenkel, Phys. Rev. A 45, 5632 (1992).

${ }^{24}$ A. Jaster, Phys. Rev. E 59, 2594 (1999), and references therein.

${ }^{25}$ M. A. Bates and D. Frenkel, to be published in Phys. Rev. E (2000).

${ }^{26}$ M. A. Bates and D. Frenkel, Phys. Rev. E 57, 4824 (1999).

${ }^{27}$ H. Schlacken, H.-J. Mögel, and P. Schiller, Mol. Phys. 93, 777 (1998).

${ }^{28}$ M. R. Fisch and C. Rosenblatt, J. Phys. II 4, 103 (1994). 